\title{
Cluster mergers, radio halos and hard X-ray tails: a statistical magneto-turbulent model
}

\author{
Rossella Cassano ${ }^{1,2}$ and Gianfranco Brunetti ${ }^{2}$ \\ ${ }^{1}$ Dip. di Astronomia, Università di Bologna \\ ${ }^{2}$ Istituto di Radioastronomia del CNR di Bologna
}

\begin{abstract}
There is now firm evidence that the ICM consists of a mixture of hot plasma, magnetic fields and relativistic particles. The most important pieces of evidence for non-thermal phenomena in galaxy clusters come from the diffuse Mpc-scale synchrotron radio emission (radio halos), observed in a growing number of massive clusters (Feretti 2003), and from hard X-ray (HXR) excess emission (detected in a few cases), which can be explained in terms of IC scattering of relativistic electrons off the cosmic microwave background photons (Fusco-Femiano et al. 2003). There is now growing evidence that giant radio halos may be naturally accounted for by synchrotron emission from relativistic electrons reaccelerated by some kind of turbulence generated in the cluster volume during merger events (Brunetti 2003). With the aim of investigating the connection between thermal and non-thermal properties of the ICM, we have developed a statistical magneto-turbulent model which describes the evolution of the thermal and non-thermal emission from clusters. We calculate the energy and spectrum of the magne!tosonic waves generated during cluster mergers, the acceleration and evolution of relativistic electrons and thus the resulting synchrotron and inverse Compton spectra. Here we give a brief description of the main results, while a more detailed discussion will be presented in a forthcoming paper (Cassano \& Brunetti, in preparation). Einstein-De Sitter cosmology, $H_{0}=50 \mathrm{~km}$ $\mathrm{s}^{-1} \mathrm{Mpc}^{-1}, q_{0}=0.5$, is assumed.
\end{abstract}

\section{Introduction}

Giovannini, Tordi and Feretti (1999) found that 5\% of clusters from a complete X-ray flux limited sample have a radio halo source. The detection rate of radio halos shows an abrupt increase with increasing X-ray luminosity and mass of the host clusters: about 30$35 \%$ of the galaxy clusters with X-ray luminosity larger than $10^{45} \mathrm{erg} \mathrm{s}^{-1}$ show diffuse non-thermal radio emission (Feretti 2003). Recent papers (Enßlin \& Röttgering 2003; Kuo, Hwang \& Ip 2003) have investigated the statistics of the formation of radio halos from a more theoretical point of view. In these works, however, the expected statistics are not derived from formation models of radio halos, but simply from the observed luminosity-mass correlations and mass thresholds.

We model the formation of radio halos and HXR tails in a self-consistent approach which follows, at the same time, the evolution of the thermal properties of the ICM and the triggering and evolution of the non-thermal phenomena by assuming magnetoturbulent re-acceleration of relativistic particles. In particular, we follow the formation and evolution of clusters of galaxies, the generation of merger-driven turbulence and magnetosonic waves in the cluster volume, the acceleration and time-evolution of the relativistic particles, and of the related non-thermal emission.

\section{The statistical magneto-turbulent model}

The major steps of the model can be sketched as follows: 

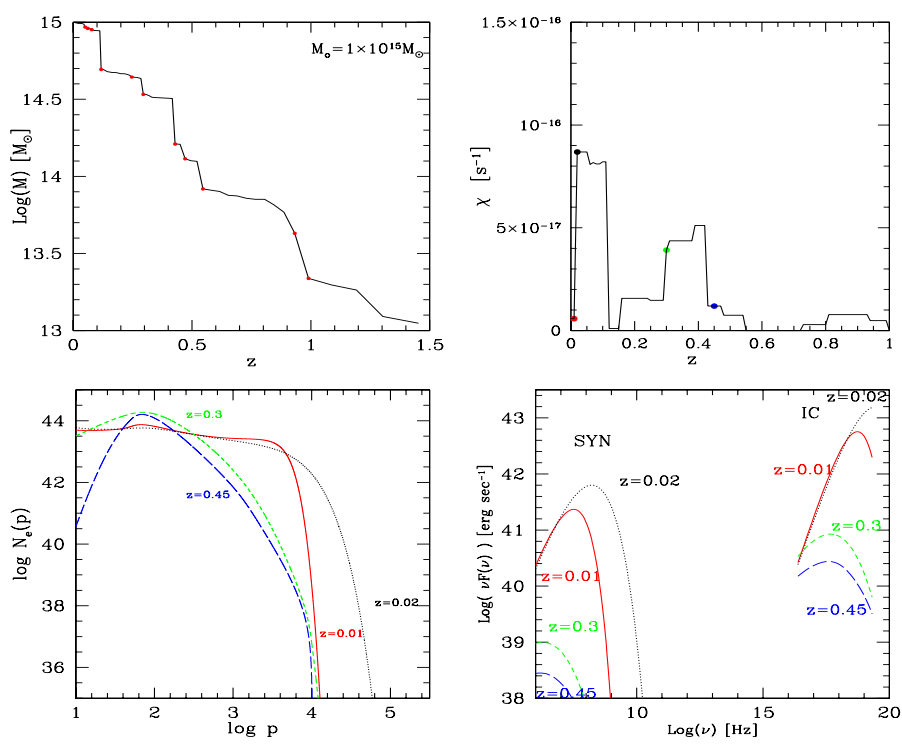

Figure 1. a) Merger-tree for a cluster with a present-day mass $M_{o}=1 \times 10^{15} M_{\odot}$; b) evolution of the electron acceleration coefficient $\left(\chi_{a c c}=\tau_{a c c}^{-1}, \tau_{a c c}\right.$ is the acceleration time) ; c) electron spectra at four different $z$; d) correspondent radio (synchrotron) and hard-X ray (IC) emission.

i) Cluster formation: The evolution and formation of galaxy clusters is computed making use of a relatively simple semi-analytic procedure based on the hierarchical Press \& Schechter (1974) theory of cluster formation. Given a present day mass and temperature of the parent clusters, the cosmological evolution (back in time) of the cluster properties (merger trees) are obtained making use of Montecarlo simulations. A suitable large number of trees allows us to describe the statistical cosmological evolution of galaxy clusters.

ii) Turbulence in galaxy clusters: The turbulence in the ICM is supposed to be injected during cluster mergers. The energetics of the turbulence is estimated from standard recipes based on the Ram Pressure Stripping theory and it is $E_{t u r} \sim \rho v_{i}^{2} \pi R_{s}^{2} R_{v}$ where $\rho$ is the density of the ICM, $v_{i}$ is the velocity between the two colliding clusters, $R_{v}$ is the virial radius of the most massive cluster and $R_{s}$ is the stripping radius (Fujita, Takizawa \& Sarazin 2003). We assume that a fraction $\eta\left(E_{M S}=\eta E_{t u r}\right)$ of the energy of the turbulence is associated to fast magneto-acoustic waves (MS waves). We use these waves since their damping rate and time evolution basically depend on the properties of the thermal plasma which are provided by our merger trees for each simulated cluster. The spectrum of the MS waves (following the injection of turbulence) is then calculated at each time step solving a turbulent-diffusion equation in the wave-number space (Eilek 1979) and assuming that the turbulence injected in the cluster volume for each merger event is injected, and thus dissipated, in a dynamical crossing time.

iii) Particle acceleration: We assume the presence of relativistic electrons in the ICM which are injected by AGNs, galactic winds, and/or merger shocks. Given the calculated spectrum of MS waves ( $i i)$ and the physical conditions in the ICM $(i)$, we compute the time evolution of relativistic electrons at each time step by solving a Fokker-Planck equation including synchrotron, IC and Coulomb losses and the effect of the electron acceleration due to the coupling between MS waves and particles.

In Fig. 1 we report an example of the results in the case of a massive cluster. The calculations are obtained assuming that the energy injected in relativistic electrons is 


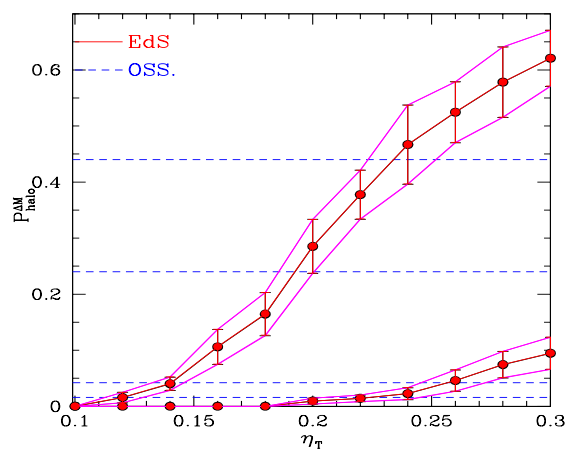

Figure 2. Formation rate of radio halos as a function of the parameter $\eta_{T}$ in two different mass bins (solid lines with error bars): $\mathrm{bin} A=[1.8-3.6] \times 10^{15} M_{\odot}$ and $\mathrm{binB}=[0.9-1.8] \times 10^{15} M_{\odot}$. The two bottom dashed lines mark the observed probabilities for radio halos in binB while the two top dashed lines mark the observed probabilities in binA.

of the order of $\sim 1 \%$ of the energy of the thermal plasma and a volume averaged $<$ $B>\sim 0.5 \mu \mathrm{G}$. The first important result is that, given these reasonable assumptions, the radio $\left(L_{R}\right)$ and hard-X ray $\left(L_{H X}\right)$ luminosities are in agreement with the observed values (typically, $L_{R} \in\left[10^{40}-10^{41}\right] \mathrm{erg} \mathrm{s}^{-1}$ and $L_{H X} \in\left[10^{42}-10^{44}\right] \mathrm{erg} \mathrm{s}^{-1}$ ).

\section{Statistics: rate of radio halos with mass}

Given a population of galaxy clusters with present day mass and temperature, combining $i$ )-iii), we follow the cosmological evolution of the non-thermal emission and the properties of the thermal ICM. We have calculated the formation rate of radio halos with cluster mass (at $z \leqslant 0.2$ ) in two bins consistent with those considerated in the observational studies: $\mathrm{bin} A=[1.8-3.6] \times 10^{15} M_{\odot}$ and $\mathrm{binB}=[0.9-1.8] \times 10^{15} M_{\odot}$. In Fig. 2 we report the formation rate of radio halos in the two mass bins as a function of the parameter $\eta\left(\eta=E_{M S} / E_{t u r}\right.$, Sect. 2). The shaded regions mark the observed range of formation rate of radio halos (Giovannini et al. 1999) in binA (top shaded region) and binB (bottom shaded region). We find that our predictions match the observations in both the mass ranges for reasonable values of $\eta \in[0.16-0.22]$. In particular, in agreement with observations, we find that $20-30 \%$ of clusters in binA can form a radio halo and that only $2-3 \%$ of galaxy clusters in binB have a radio halo.

\section{References}

Brunetti, G. 2003, in Matter and Energy in Clusters of Galaxies, p.349, ASP, Conf. Ser. v.301, eds: S.Bowyer \& C-Y.Hwang.

Eilek, A.J. 1979, ApJ 230, 373.

Enßlin T.A., Röttgering H. 2002, A\&A 396, 83.

Feretti L. 2003, in Matter and Energy in Clusters of Galaxis, p.143, ASP Conf. Ser. v.301, eds: S.Bowyer \& C-Y.Hwang.

Fujita Y., Takizawa M., Sarazin C.L. 2003, ApJ 584, 190.

Fusco-Femiano R., Dal Fiume D., Orlandini M., et al. 2003, in Matter and Energy in Clusters of Galaxies, p.109, ASP, Conf. Ser. v.301, eds: S.Bowyer \& C-Y.Hwang.

Giovannini G., Tordi M., Feretti L. 1999, New Astronomy 4, 141.

Kuo P-H., Hwang C-Y., Ip W-H. 2004, ApJ 604,108.

Press W.H., Schechter P. 1974, ApJ 187, 425. 